\title{
The role of $T$ cell subsets and cytokines in the regulation of intracellular bacterial infection
}

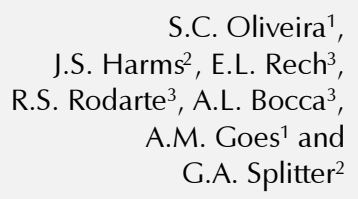

S.C. Oliveira ${ }^{1}$, J.S. Harms ${ }^{2}$, E.L. Rech', R.S. Rodarte ${ }^{3}$, A.L. Bocca ${ }^{3}$, A.M. Goes ${ }^{1}$ and G.A. Splitter ${ }^{2}$

\author{
'Departamento de Bioquímica e Imunologia, Universidade Federal de Minas Gerais, \\ Belo Horizonte, MG, Brasil \\ ${ }^{2}$ Department of Animal Health and Biomedical Sciences, University of Wisconsin, \\ Madison, WI, USA \\ ${ }^{3}$ Centro Nacional de Pesquisa de Recursos Genéticos e Biotecnologia-EMBRAPA, \\ Brasília, DF, Brasil
}

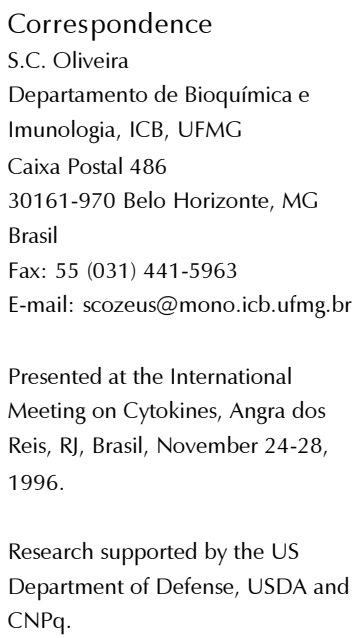

Received September 24, 1997 Accepted September 30, 1997

\begin{abstract}
Cellular immune responses are a critical part of the host's defense against intracellular bacterial infections. Immunity to Brucella abortus crucially depends on antigen-specific $T$ cell-mediated activation of macrophages, which are the major effectors of cell-mediated killing of this organism. T lymphocytes that proliferate in response to B. abortus were characterized for phenotype and cytokine activity. Human, murine, and bovine $\mathrm{T}$ lymphocytes exhibited a type 1 cytokine profile, suggesting an analogous immune response in these different hosts. In vivo protection afforded by a particular cell type is dependent on the antigen presented and the mechanism of antigen presentation. Studies using MHC class I and class II knockout mice infected with B. abortus have demonstrated that protective immunity to brucellosis is especially dependent on $\mathrm{CD} 8+\mathrm{T}$ cells. To target $\mathrm{MHC}$ class I presentation we transfected ex vivo a murine macrophage cell line with $\mathrm{B}$. abortus genes and adoptively transferred them to BALB/c mice. These transgenic macrophage clones induced partial protection in mice against experimental brucellosis. Knowing the cells required for protection, vaccines can be designed to activate the protective $\mathrm{T}$ cell subset. Lastly, as a new strategy for priming a specific class I-restricted $T$ cell response in vivo, we used genetic immunization by particle bombardment-mediated gene transfer.
\end{abstract}

\section{Introduction}

Intracellular bacteria are microbial pathogens which share several common features and include a variety of organisms such as Listeria monocytogenes, Salmonella typhimurium, Mycobacterium tuberculosis and Brucella abortus. Optimal protective immunity to intracellular bacteria usually requires the combination of different elements of the immune system to orchestrate cellular responses leading to protection. To be more
Key words

- T cell subsets

- Brucella abortus

- Knockout mice

- Cytokines

- Intracellular pathogens

- Genetic immunization 
stable subsets according to their use of $\mathrm{T}$ cell receptors, accessory molecules that interact with the MHC gene products on target cells, and cytokine profile. In the present review we stress the role of $\mathrm{CD} 4+$ and $\mathrm{CD} 8+\alpha \beta \mathrm{T}$ cells in the regulation of infection. Immunity to intracellular bacteria was considered to be exclusively mediated by CD4+ T cells (1). Recently, optimal protection against intracellular bacteria is regarded as a coordinated interaction between different $T$ cell subsets (2). Additional studies have evoked a central role for CD8 + $\mathrm{T}$ lymphocytes against intracellular pathogens $(3,4)$. However, the principal mechanism by which CD8+ T cells coordinate antimicrobial resistance remains to be determined. Here, we propose that the combination of different functions of $\mathrm{CD} 8+\mathrm{T}$ cells such as IFN- $\gamma$ production, cytotoxic activity, and down-regulation of type 2 cytokines is pivotal to control intracellular infection.

This review describes experiments performed with Brucella abortus, a facultative intracellular bacterium, to illustrate i) that Brucella abortus induces a type 1 cytokine profile in murine, bovine, and human $T$ lymphocytes, ii) the role of $\mathrm{CD} 4+$ and $\mathrm{CD} 8+\mathrm{T}$ lymphocytes in controlling infection, iii) that a transfected macrophage cell line presents Brucella peptides in the context of MHC class I molecules, and iv) genetic immunization as a new strategy to induce $C D 8+T$ cellmediated protection.

Brucella abortus is a Gram-negative facultative intracellular bacterium that infects humans and domestic animals. The pathological manifestations of brucellosis are diverse and include arthritis, endocarditis, undulant fever and meningitis in humans, while bovine brucellosis is characterized by spontaneous abortion (5). Brucella replicates in host mononuclear phagocytes, and survival in phagocytic cells allows the bacterium to escape the extracellular mechanisms of host response such as complement and antibodies. Therefore, T lymphocyte activation upon vaccination with bacterial proteins appears to be a decisive cell-mediated mechanism to provide resistance to $\mathrm{B}$. abortus infection.

Pathogenesis induced by intracellular organisms is the product of a complex series of interactions between the pathogen, the infected host cell, and different elements of the immune system. Thus, research on the interplay between the immune response and persistent microbial pathogens as well as the characterization of the relevant antigens will help us to understand how the immune system functions against different diseases.

Brucella abortus induces a type 1 cytokine profile in murine, bovine, and human $\mathrm{T}$ lymphocytes

Cytokines are key molecules that play a major role in determining a protective or a noncurative immune response. Initially, $\mathrm{CD} 4+\mathrm{T}$ cells were divided into at least two subsets upon antigen stimulation and cytokine production (6). The ability of specific antigens to induce preferentially a Th1 or Th2 (T-helper) subset response is an important aspect for the development of vaccines against intracellular pathogens (7). These distinct $T$ cell subsets often influence the outcome of infection through the production of specific cytokines. Currently, the type 1 or type 2 cytokine concept has been extended to other cell types such as CD8 $+\alpha \beta$ T cells and $\gamma \delta \mathrm{T}$ lymphocytes (8). The objective of this section is to compare the cytokine profiles induced by B. abortus infection in different hosts. The pattern of cytokines reported here was detected using quantitative RT-PCR for transcription profiles or ELISA for secreted products.

Understanding the influence of cytokines produced by the innate immune system on the acquisition of adequate $T$ cell responses to an infectious agent is critical to define the pathway of Th or Tc (T cytotoxic) cell development. It is now becoming clear that the divergence into Th1 and Th2 cells is regulated to some extent by the innate immune response at the onset of infection. IL-12 and IL-4 have been identified as early inducers 
of Th1 or Th 2 cell responses, respectively (9). Recently, investigators have demonstrated that human monocytes expressed mRNA transcripts for IL-12 p40 at $4 \mathrm{~h}$ and secreted IL-12 p40 protein at $24 \mathrm{~h}$ after in vitro stimulation with $\mathrm{B}$. abortus (10). We also demonstrated that adherent murine spleen cells showed an elevated IL-12 p40 gene expression level at 12 to $24 \mathrm{~h}$ after stimulation with $\gamma$-irradiated B. abortus, returning to normal expression levels after 48 $\mathrm{h}$ (Oliveira SC and Splitter GA, unpublished results). This early production of IL-12 during $\mathrm{B}$. abortus infection is pivotal to activate the pathway of IFN- $\gamma$-producing Th1 cells.

In the bovine model, Brucella-primed peripheral blood mononuclear cells (PBMC) showed an elevation of transcripts for IFN- $\gamma$ but not for IL-2 or IL-4 when compared to PBMC from naive animals (Covert J and Splitter GA, unpublished results). An identical profile is exhibited by mouse splenocytes and purified CD4+ and CD8+ T lymphocytes from B. abortus-infected animals $(11,12)$. In the murine system, the type 1 cytokine profile was confirmed by detection of IFN- $\gamma$ but not IL-2 or IL-4 in the cell supernatants. A strong correlation between the level of cytokine mRNA transcripts and the cytokine pattern secreted by $\mathrm{T}$ lymphocytes has been established.Additionally, B. abortus is a $\mathrm{T}$ cell-independent antigen which has been used as a carrier for different human vaccines, such as HIV and malaria (13). In concordance with our studies, others have demonstrated that B. abortus induced IFN- $\gamma$ secretion by purified human CD4+ and CD8 + T cells, indicating the ability of this bacterium to promote a Th1 pattern of $\mathrm{T}$ cell differentiation (14). The cytokine transcription profile as well as secreted products are closely similar among murine, bovine, and human $\mathrm{T}$ cells upon $\mathrm{B}$. abortus stimulation (15). Identical immune responses mounted by these different species against the same pathogen reinforce the use of animal models to study human diseases and the applicability of immunological information to these species.
The role of CD4 + and $C D 8+T$ cells in controlling infection

Earlier murine brucellosis studies indicated the importance of $T$ cell subsets in the resolution of infection but produced conflicting data, suggesting that CD4+ and/or CD8 + $\mathrm{T}$ cells were responsible for protection (16). The paradigm of disease resolution was based on adoptive $T$ cell transfer or in vivo subpopulation depletion using monoclonal antibodies, both with potential pitfalls. To clarify the controversy about the $T$ cell subsets responsible for B. abortus disease resolution, we have used gene knockout (KO) mice. Research into anti-infectious immunity has enormously benefited from the creation of KO mice by homologous recombination which has not only clarified the essential role of distinct immune components, but also unraveled an extraordinary compensatory capacity of the system. Mice in which the B2-microglobulin gene (MHC class I KO) was disrupted or the MHC class II $\alpha \beta$ chain (MHC class II KO) is nonfunctional are devoid of functional $\mathrm{CD} 8+$ or CD4+ T cells, respectively. Using MHC class $\mathrm{I}$ and class II $\mathrm{KO}$ mice and $\mathrm{C} 57 \mathrm{BL} / 6$ as controls, CD8 $+\mathrm{T}$ cells were found to be critical in resolving B. abortus infection. Moreover, our findings provide insights into both effector and regulatory mechanisms used by CD $8+T$ cells to control intracellular bacterial infection.

To determine the contribution of CD4+ or CD8+ T cells to the control of brucellosis, bacterial numbers were monitored in spleens of MHC class I KO, MHC class II KO, and C57BL/6 mice for four weeks following infection. The animals were sacrificed weekly and $\mathrm{B}$. abortus colony-forming units (CFU) were counted. Interestingly, murine brucellosis was markedly exacerbated in animals lacking functional CD8+ T cells (MHC class I KO) compared to MHC class II $\mathrm{KO}$ or C57BL/6 control mice. After the first week post-infection, B. abortus CFU increased in 
the spleens of MHC class I KO mice whereas MHC class II KO mice displayed a constant decline in bacterial numbers throughout the course of infection. Additionally, the number of B. abortus CFU in MHC class I KO mouse spleens was at least two logs higher than that observed in spleens of MHC class II-deficient mice during the four weeks postinfection. This result clearly demonstrates the impact of CD8 $+\mathrm{T}$ cells on cell-mediated immunity in murine brucellosis (12). Although MHC class I deficiency had much more dramatic consequences for $\mathrm{B}$. abortus replication than the lack of MHC class II molecules, $\mathrm{CD} 4+\mathrm{T}$ cells are likely to play a helpful but not essential role in the protection against $\mathrm{B}$. abortus infection.

The cytokine transcription profile and the secreted products were assayed to determine which of these important regulatory molecules are involved in orchestrating the cellular immune response in brucellosis. The results indicated that Brucella-primed splenocytes from all three mouse strains exhibited a type 1 cytokine profile (12). Up-regulation of mRNA transcripts for IFN- $\gamma$, but not for IL-2 or IL-4, was detected. C57BL/6 and MHC class I KO mouse splenocytes displayed a ten-fold increase in the level of IFN- $\gamma$ mRNA expression compared to splenocytes from naive animals. Interestingly, MHC class II KO mouse splenocytes showed only a two-fold elevation of IFN- $\gamma$ transcripts within the first two weeks after B. abortus infection. However, IFN- $\gamma$ transcription in
MHC class II KO mouse splenocytes reached levels similar to those of the other strains after two weeks post-infection. To confirm that the transcriptionally active cytokines were also secreted by antigen-activated spleen cells, IL-2, IL-4, and IFN- $\gamma$ were determined by ELISA. IFN- $\gamma$ was secreted by spleen cells from all mouse strains when the cells were cultured with $\gamma$-irradiatedB. abortus. To elucidate the cytokine network involved in murine brucellosis and its possible role in enhancing resistance or susceptibility to B. abortus, we measured IL-10 and TGFB1 in splenocyte culture supernatants by ELISA. IL-10 and TGF- $\beta 1$ are cytokines known to be involved in down-regulating protective immune responses by inhibiting Th1 cell differentiation or by blocking cytokine-induced macrophage activation (17). We did not detect production of TGF- $\beta 1$ by splenocytes from any of the three mouse strains studied following B. abortus infection. In contrast, substantial amounts of IL10 were detected in splenic culture supernatants from all three mouse strains. Surprisingly, spleen cells from MHC class I KO mice lacking functional $\mathrm{CD} 8+\mathrm{T}$ cells produced two-fold higher levels of IL-10 than splenocytes from MHC class II KO or C57BL/6 mice. We correlated the enhanced susceptibility of MHC class I KO mice to brucellosis with the ability of MHC class I $\mathrm{KO}$ spleen cells to produce higher levels of IL-10. According to our data and recent findings reported by others, we hypothesize
Figure 1 - Analysis of cytokine gene expression in B. abortusprimed and naive $C D 8+T$ cells using a competitive RT-PCR-based assay. Spleen cells were separated from MHC class II KO and C57BL/6 naive mice before and one week after infection by cell sorting. We obtained $97 \%$ pure CD8+ T cells and extracted their RNA. The RT-PCR products were quantified in comparison to competitive internal standards for IL-2, IL-4 and IFN- $\gamma$ using a gel video system (NIH image 1.54) to scan and quantify ethidium bromidestained gel bands. The results are reported as attomoles.

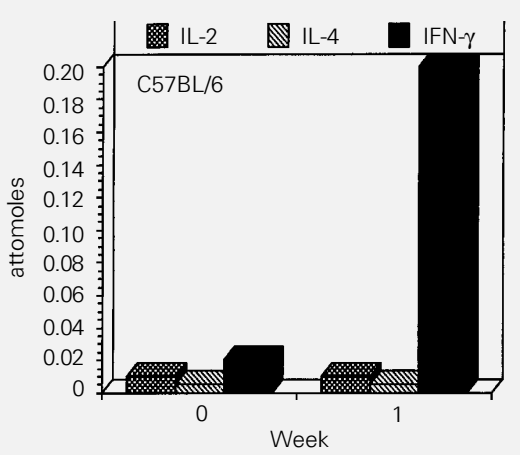




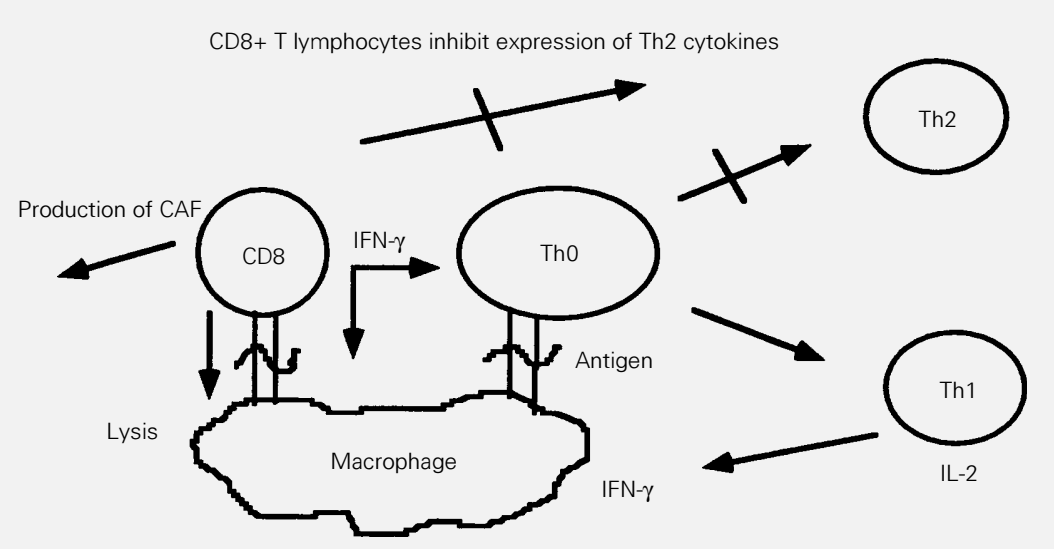

that $\mathrm{CD} 8+\mathrm{T}$ cells may inhibit IL-10 production and, as a consequence, murine brucellosis was exacerbated in MHC class I KO mice (18). IL-10 production byBrucella-activated macrophages may be associated with a high concentration of suppressor macrophages found in mouse spleens two to three weeks after infection.

To better define the mechanisms used by CD8+ T cells to control B. abortus infection, a cytokine transcription profile of this purified $\mathrm{T}$ cell subpopulation was determined. CD8+ T cells from B. abortus-infected MHC class II $\mathrm{KO}$ or C57BL/6 mice demonstrated no increase in mRNA expression for IL-2 or IL-4 when compared to CD8+ T cells from untreated mice. However, IFN- $\gamma$ mRNA was markedly elevated in CD8 $+\mathrm{T}$ cells from both B. abortus-infected mouse strains when compared to naive groups (Figure 1). The higher levels of IFN- $\gamma$ expression observed in $\mathrm{MHC}$ class II KO CD8+ T cells compared to $\mathrm{MHC}$ class I $\mathrm{KO} \mathrm{CD} 8+\mathrm{T}$ lymphocytes might serve as a compensatory mechanism for the lack of CD4+ T cells. Thus, Brucellaprimed CD8+ T cells from MHC class II KO or C57BL/6 mice exhibited a type 1 cytokine profile identical to the pattern displayed by unseparated spleen cells from infected animals. IFN- $\gamma$ plays a central role in acquired resistance of mice against intracellular bacteria. This cytokine is known to up-regulate macrophage microbial killing activity and to induce the development of Th1 cells. There- fore, the production of IFN- $\gamma$ by CD8 $+\mathrm{T}$ cells seems to be one of the important mechanisms by which this $T$ cell subpopulation helps the immune system to control infection. CD8+ T cells can be activated early in the immune response and they are in an ideal position to regulate $\mathrm{CD} 4+\mathrm{T}$ cell development by producing IFN- $\gamma$ or other regulatory cytokines. Regarding effector function, B. abortus CD8+ T cells from MHC class II KO or C57BL/6 mice specifically lysed MHC class II KO- or C57BL/6-infected splenic macrophages, respectively (12).

Our studies provide unequivocal data demonstrating the central role played by CD8 $+\mathrm{T}$ cells in controlling an intracellular bacterial infection. In Figure 2 we propose a model of the way CD8 $+\mathrm{T}$ cells function in disease resolution. We suggest that a combination of mechanisms underlie the acquisition of protective immunity against this intracellular bacterium. First, CD8 $+\mathrm{T}$ cells exhibited a type 1 cytokine profile, marked by IFN- $\gamma$ production, a critical cytokine involved in macrophage activation and Th1 cell development. Second, mice lacking CD8+ T cells produced two-fold higher levels of IL-10, an immunosuppressive cytokine, than the other mouse strains evaluated. These data corroborate recent findings reported by other investigators showing that CD8+ $\mathrm{T}$ cells down-regulate expression of Th2 cytokines such as IL-10 (18). Third, primed CD8+ $\mathrm{T}$ cells were able to lyse Brucella-infected
Figure 2 - Schematic representation of the role of CD8+ T lymphocytes in controlling B. abortus infection. The roles are i) production of IFN- $\gamma$, ii) lysis of Brucella-infected macrophages, iii) down-regulation of Th2 cytokines such as IL-10, and iv) production of a new cytokine-like $\mathrm{CD} 8+\mathrm{T}$ cell antiviral factor (CAF). 
macrophages, and finally, CD8+ T cells may produce other soluble factors or cytokines such as $\mathrm{CD} 8+\mathrm{T}$ cell antiviral factor in the HIV model which are important to regulate infection (19).

A transfected macrophage cell line presents Brucella peptide in the context of MHC class I molecules

Our results have emphasized a major role of MHC class I-restricted CD8+ T cells in the protection against intracellular bacteria. MHC class I molecules primarily present endogenous antigens that are synthesized by antigen-presenting cells and processed within the cytosol. After cytosolic delivery, proteosomes are proposed to cleave antigens into peptides, which are then transported into the lumen of the endoplasmic reticulum (ER) via a transporter for antigen presentation. In the ER, peptides bind to nascent MHC class I molecules which can then be transported to the cell surface. Therefore, translocation of Brucella antigens from the endosome into the cytosol is required for the stimulation of Brucella-specific MHC class I restricted CD8+ T cells. Interestingly, B. abortus strain 2308 inhibits phagosome-lysosome fusion and can be found in the ER of infected nonphagocytic cells, which would be an optimal localization for presentation through the MHC class I pathway.

We have identified different B. abortus genes and produced their respective immunodominant products that activate Brucellaprimed CD4+ $\mathrm{T}$ lymphocytes in vitro (20). In an attempt to shift the peptide presentation pathway from an endocytic to a cytosolic one and to induce antigen-specific CD8+ $\mathrm{T}$ cell activation, we transfected Brucella genes into a murine macrophage-like tumor cell line (Raw 264.7). First, we subclonedB. abortus GroES, GroEL, uvrA, and L7/L12 genes known to encode immunodominant products into a mammalian expression vector (pCDNA3). These constructs were then stably transfected into Raw 264.7 cells. To confirm gene expression, macrophage clones expressing Brucella genes were screened by Northern blot using the respective probes. Macrophage clones transfected with Brucella genes were then cultured in the presence of enriched murine Brucella-primed $T$ lymphocytes to measure $T$ cell proliferative responses. Additionally, medium alone or monoclonal antibodies (mAbs) to an irrelevant antigen, anti-KD ${ }^{\mathrm{d}}$ (MHC class I), antiCD4 or anti-CD8 molecules, were added to the culture system. When medium alone or $\mathrm{mAb}$ to an irrelevant antigen or anti-CD4 $\mathrm{mAb}$ was added, primed $\mathrm{T}$ lymphocytes proliferated to macrophages transfected with GroEL or L7/L12 genes. However, when anti-CD8 or anti-KD ${ }^{\mathrm{d}} \mathrm{mAb}$ was included, the proliferation was abrogated to background levels. Using this methodology we showed that antigen-specific recognition ofBrucella GroEL and L7/L12 peptides was restricted to MHC class I molecules and dependent upon $\mathrm{CD} 8+\mathrm{T}$ cells. Therefore, using this system we can target MHC class I presentation of specific Brucella peptides.

To investigate if these transgenic macrophage clones indeed induce protective immunity in mice, the transfected Raw 264.7 cells were $\gamma$-irradiated and adoptively transferred to syngeneic BALB/c mice weekly at a concentration of $1 \times 10^{6}$ cells. Surprisingly, Raw 264.7 cells transfected with the Brucella GroEL or L7/L12 gene induced partial protective immunity of BALB $/ \mathrm{c}$ mice against B. abortus infection (Oliveira SC, Harms JS and Splitter GA, unpublished results). These findings are consistent with the importance of engaging the endogenous antigen-presenting pathway to bias the immune response towards a CD8 $+\mathrm{T}$ lymphocyte activation during an intracellular bacterial infection.

Genetic immunization as a new strategy to induce CD8+ $\mathrm{T}$ cell-mediated protection

Traditionally, classical vaccines have been produced by the use of attenuated or 
inactivated pathogens and immunogenic protein subunits of microbes. A novel vaccine approach, originally developed for gene therapy, is based on immunization with DNA or RNA molecules. Tang et al. (21), who first described the induction of an immune response upon inoculation of plasmid vectors, referred to this approach as genetic immunization, a nomenclature that will be used throughout this review. Direct inoculation of the gene(s) of a pathogen should mimic attenuated vaccines since synthesis of specific foreign proteins would be achieved in the host and would be the target of immune surveillance. The understanding of the different pathways for antigen presentation led to the consideration of direct transfection of tissue cells in vivo as an alternative means of obtaining appropriate antigen processing, and thus as an alternative to immunization with live virus, for example. Genetic immunization is a simple and efficient technique to sensitize $T$ cells. It involves the injection into animals of expression plasmid DNA that encodes the antigen of interest. Transient expression of the DNA in an antigenpresenting cell in the skin, muscle, endothelium or vascular smooth muscle allows the priming of immune responses. DNA immunization has been reported to successfully induce MHC class I-restricted cytotoxic T lymphocyte activity against different pathogens in many different animal models (22). In addition, strong and long-lasting humoral and cellular immune responses are also obtained (23). This novel vaccination approach has been shown to confer protection against influenza virus, rabies virus, hepatitis B virus, malaria, leishmaniasis, and tuberculosis, among others (24).

There are basically two main DNA delivery methods: direct injection into skeletal muscle using a syringe and a small gauge needle and particle bombardment or biolistic process using a gene gun device. Here, we discuss our preliminary data showing induction of an immune response to $\beta$-galactosidase as a reporter protein using biolistic-

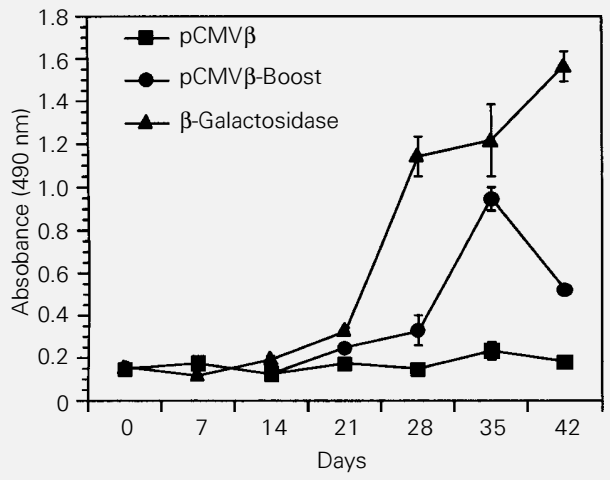

mediated gene transfer (25). Plasmid $\mathrm{pCMVß}$ containing the human cytomegalovirus (CMV) immediate early enhancer/promoter fused to theEscherichia coli $\beta$-galactosidase gene was used in this experiment. Gold microparticles $(1.5-3.0 \mu \mathrm{m})$ were coated with plasmid DNA and introduced into the skin of $\mathrm{BALB} / \mathrm{c}$ mice using a hand-held high pressure helium-driven device. The ear and peritoneal regions were the target for gene gunbased DNA immunization in all animals. For each bombardment we used $1 \mu \mathrm{g}$ of DNA and the boost was performed 15 days after the first immunization. Figure 3 shows a strong elevation of total IgG titers against $B$ galactosidase in mice vaccinated with pCMVß after the boost compared to the levels prior to immunization. Mice immunized with $\beta$-galactosidase alone were used as a positive control in our vaccination protocol. We are currently in the process of measuring cell-mediated immune responses by $T$ cell proliferation, cytokine profile analysis and cytotoxic activity to $ß$-galactosidase after DNA immunization. The $\beta$-galactosidase gene was used to evaluate the physical parameters to introduce and express exogenous DNA. Now, we are in the position to test the ability of Brucella-specific genes such as GroEL and L7/L12 in conferring protective immunity by the biolistic process. Using this methodology, we intend to better activate specific CD8 $+\mathrm{T}$ cells targeting the MHC class I presentation pathway. This novel approach is a potent strategy for priming
Figure 3 - Total IgG anti-ß-galactosidase titers were determined by ELISA in sera from three groups of BALB/c mice which were immunized by biolistic-mediated gene transfer using DNA or protein. The first group of five mice was vaccinated with the pCMVß construct (squares), the second group received the same treatment with a boost 15 days after the first immunization (circles), and the third group was immunized with $10 \mu \mathrm{g}$ of the protein ß-galactosidase (triangles). Microtiter plates were coated overnight with $10 \mu \mathrm{g} / \mathrm{ml}$ ß-galactosidase and serum samples from mice $(1: 200)$ were incubated for 2 $\mathrm{h}$ at room temperature. After washing, the plates were incubated with goat anti-mouse lgG peroxidase conjugate for $2 \mathrm{~h}$. The reaction was developed and absorbance was read at $490 \mathrm{~nm}$. 
class I-restricted $\mathrm{T}$ cell responses in vivo, and is important for future vaccine development. Also, it is particularly relevant for intracellular microbial pathogens, none of which can be satisfactorily hindered by conventional vaccination strategies at present.

\section{References}

1. Zinkernagel RM (1974). Restriction by H-2 gene complex of transfer of cell-mediated immunity to Listeria monocytogenes. Nature, 251: 230-233.

2. Ladel $\mathrm{CH}$, Flesch IEA, Arnoldi J \& Kaufmann SH (1994). Studies with MHCdeficient knock-out mice reveal impact of both MHC-I and MHC-II dependent T cell responses on Listeria monocytogenes infection. Journal of Immunology, 153: 3116-3122.

3. Flynn JL, Goldstein MM, Triebold KJ, Koller B \& Bloom BR (1992). Major histocompatibility complex class I-restricted $T$ cells are required for resistance to $\mathrm{Myco}-$ bacterium tuberculosis infection. Proceedings of the National Academy of Sciences, USA, 89: 12013-12017.

4. Harty JT, Schreiber RD \& Bevan MJ (1992). CD8 T cells can protect against an intracellular bacterium in an interferon gamma-independent fashion. Proceedings of the National Academy of Sciences, USA, 89: 11612-11616.

5. Nicoletti PL (1989). Relationship between animal and human disease. In: Young EJ \& Corbel MJ (Editors), Brucellosis: Clinical and Laboratory Aspects. CRC Press, Inc., Boca Raton, FL.

6. Mosmann TR, Cherwinski H, Bond MN, Giedlin MA \& Coffman RL (1986). Two types of murine helper $\mathrm{T}$ cell clone: 1 . Definition according to profiles of lymphokine activities and secreted protein. Journal of Immunology, 136: 2348-2357.

7. Mosmann TR \& Coffman RL (1987). Two types of mouse helper T cell clone: Implications for immune regulation. Immunology Today, 8: 223-227.

8. Kemeny DM, Noble A, Holmes BJ \& DiazSanchez D (1994). Immune regulation: a new role for the CD8+ T cell. Immunology Today, 15: 107-110.

9. Kaufmann SH (1995). Immunity to intracellular microbial pathogens. Immunology Today, 16: 338-342.

10. Zaitseva M, Golding H, Manischewitz J, Webb D \& Golding B (1996). Brucella abortus as a potential vaccine candidate: induction of IL-12 secretion and enhanced B7-1 and B7-2 and ICAM-1 surface expression in elutriated human monocytes stimulated by heat-inactivated $B$. abortus. Infection and Immunity, 64: 3109-3117.

11. Oliveira SC, Zhu Y \& Splitter GA (1994). Recombinant L7/L12 ribosomal protein and $\gamma$-irradiated Brucella abortus induce a T-helper 1 subset response from murine CD4+ T cells. Immunology, 83: 659-664.

12. Oliveira SC \& Splitter GA (1996). CD8+ type 1 CD44hiCD45Rblo $T$ lymphocytes control intracellular Brucella abortus infection as demonstrated in major histocompatibility complex class I- and class IIdeficient mice. European Journal of Immunology, 25: 2551-2557.

13. Lapham C, Golding B, Inmann J, Blackburn R, Manischewitz J, Highet P \& Golding H (1996). Brucella abortus conjugated with a peptide derived from the V3 loop of human immunodeficiency virus (HIV) type 1 induces HIV-specific cytotoxic T-cell responses in normal and in CD4+ $T$ cell-depleted BALB/c mice. Journal of $\mathrm{Vi}$ rology, 70: 3084-3092.

14. Zaitseva MB, Golding $H$, Betts $M$, Yamaauchi A, Bloom ET, Butler LE, Stevan L \& Golding B (1995). Human peripheral blood CD4+ and CD8+ $T$ cells express Th1-like cytokine mRNA and proteins following in vitro stimulation with heat-inactivated Brucella abortus. Infection and Immunity, 63: 2720-2728.

15. Splitter G, Oliveira S, Carey M, Miller C, Ko J \& Covert J (1996). T Iymphocyte mediated protection against facultative intracellular bacteria. Veterinary Immuno/ogy and Immunopathology, 54: 309-319.

16. Araya $L N$, Elzer $P$, Rowe GE, Enright FM \& Winter AJ (1989). Temporal development of protective cell-mediated and humoral immunity in BALB/c mice infected with Brucella abortus. Journal of Immunology, 143: 3330-3337.

17. Fiorentino DF, Zlotnik $A$, Vieira $P$, Mosmann TR, Howard M, Moore KW \& O'Garra A (1991). IL-10 acts on the antigen-presenting cell to inhibit cytokine pro- duction by Th1 cells. Journal of Immunology, 146: 3444-3451.

18. Noble A, Staynov DZ, Diaz-Sanchez D, Lee TH \& Kemeny DM (1993). Elimination of IgE regulatory rat $\mathrm{CD} 8+\mathrm{T}$ cells in vivo increases the co-ordinate expression of Th2 cytokines IL-4, IL-5 and IL-10. Immunology, 80: 326-329.

19. Levy JA, Mackewicz CE \& Barker E (1996). Controlling HIV pathogenesis: the role of the noncytotoxic anti-HIV response of CD8+ T cells. Immunology Today, 17: 217-224.

20. Oliveira SC, Harms JS, Banai M \& Splitter GA (1996). Recombinant Brucella abortus proteins that induce proliferation and gamma-interferon secretion by $\mathrm{CD} 4+\mathrm{T}$ cells from Brucella-vaccinated mice and delayed-type hypersensitivity in sensitized guinea pigs. Cellular Immunology, 172: 262-268.

21. Tang D, DeVit M \& Johnston SA (1992). Genetic immunization is a simple method for eliciting an immune response. Nature, 356: 152-154.

22. Kuhober $A$, Pudollek H-P, Reifenberg $K$, Chisari FV, Schlicht H-J, Reimann J \& Schirmbeck R (1996). DNA immunization induces antibody and cytotoxic $T$ cell responses to hepatitis $B$ core antigen in $\mathrm{H}$ $2^{\mathrm{b}}$ mice. Journal of Immunology, 156: 3687-3695.

23. Robinson $\mathrm{HL}$, Hunt LA \& Webster RG (1993). Protection against a lethal influenza virus challenge by immunization with a haemagglutinin expressing plasmid DNA. Vaccine, 11: 957-960.

24. Davis $\mathrm{HL}$, Mancini $M$, Michel M-L \& Whalen RG (1996). DNA mediated immunization to hepatitis B surface antigen: longevity of primary response and effect of boost. Vaccine, 14: 910-915.

25. Rech EL, De-Bem AR \& Aragão FJL (1996). Biolistic-mediated gene expression in guinea pigs and cattle tissues in vivo. Brazilian Journal of Medical and Biological Research, 29: 1265-1267. 